\title{
RESEARCH
}

Open Access

\section{Melatonin rescued interleukin $1 \beta$-impaired chondrogenesis of human mesenchymal stem cells}

\author{
Bo Gao ${ }^{1 \dagger}$, Wenjie Gao ${ }^{2,4,5 \dagger}$, Zizhao Wu ${ }^{1 \dagger}$, Taifeng Zhou ${ }^{2,4}$, Xianjian Qiu' ${ }^{1}$ Xudong Wang ${ }^{1}$, Chengjie Liann ${ }^{2,4}$,

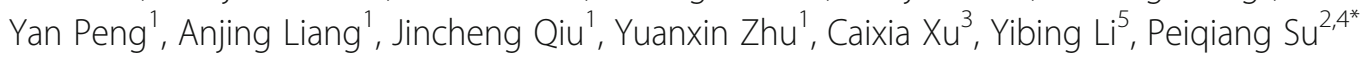 \\ and Dongsheng Huang ${ }^{1 *}$ (D)
}

\begin{abstract}
Background: Osteoarthritis $(\mathrm{OA})$ is a widespread arthritic disease and a primary cause of disability. Increasing

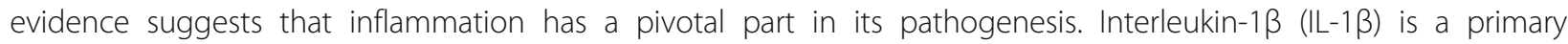
mediator of local inflammatory processes in OA. Current therapies for OA mainly focus on the symptoms of the advanced stage of the disease. The possible utilization of bone marrow mesenchymal stem cells (BMSCs) to regenerate cartilage is an appealing method, but in the case of OA requires chondrogenesis to take place within an inflamed environment. Our previous study showed that melatonin (MLT) can promote chondrogenic differentiation of MSCs, but whether MLT can rescue IL-1 $\beta$-impaired chondrogenesis in human BMSCs has not yet been established. MLT, which can have anti-inflammatory and prochondrogenic effects, has demonstrated potential in defeating IL-1 $\beta$-induced inhibition of chondrogenesis and further study should be conducted.

Methods: Human bone marrow-derived MSCs were separated and cultured based on our system that was already documented. A high-density micromass culture system was used for the chondrogenic differentiation of human BMSCs, which was also described previously. Human BMSCs were induced for chondrogenesis for 7, 14, and 21 days with the treatment of IL-1 $\beta$ and MLT. The cultured cartilage pellets were then evaluated by morphology, extracellular matrix accumulation, and chondrogenic, metabolic, and apoptotic marker expression. Furthermore, cell apoptosis was assessed by TUNEL assay. The phosphorylation level P65 and IKBa of the NF-kB pathway activity was explored on day 21 of chondrogenic differentiation of BMSCs.

(Continued on next page)
\end{abstract}

\footnotetext{
*Correspondence: supq@mail.sysu.edu.cn; huangdongshen18@hotmail.com

${ }^{+}$Bo Gao, Wenjie Gao and Zizhao Wu contributed equally to this work.

2Department of Orthopedics, The First Affiliated Hospital of Sun Yat-sen

University, Guangzhou, Guangdong, China

${ }^{1}$ Department of Orthopedics, Sun Yat-sen Memorial Hospital of Sun Yat-sen

University, \#107 West Yan Jiang Road, Guangzhou 510120, Guangdong,

China

Full list of author information is available at the end of the article
}

(c) The Author(s). 2018 Open Access This article is distributed under the terms of the Creative Commons Attribution 4.0 International License (http://creativecommons.org/licenses/by/4.0/), which permits unrestricted use, distribution, and reproduction in any medium, provided you give appropriate credit to the original author(s) and the source, provide a link to the Creative Commons license, and indicate if changes were made. The Creative Commons Public Domain Dedication waiver (http://creativecommons.org/publicdomain/zero/1.0/) applies to the data made available in this article, unless otherwise stated. 
(Continued from previous page)

Results: The current evaluation showed that MLT can save IL-1 $\beta$-impaired chondrogenesis of human BMSCs in different aspects. Firstly, MLT can restore the chondrogenic pellet size, and rescue matrix synthesis and accumulation. Secondly, MLT can upregulate chondrogenic marker COL2A1 expression at both mRNA and protein levels, and also regulate the expression levels of other chondrogenic markers like ACAN, SOX9, and COL10A1 in the presence of IL-1 $\beta$. Thirdly, MLT can maintain the metabolic balance of the chondrogenic process by suppressing expression of catabolic genes, such as MMP, MMP13, and ADAMTS4. Furthermore, MLT can subdue IL-1 $\beta$-induced cell apoptosis of BMSCs throughout chondrogenesis. Meanwhile, MLT suppressed the phosphorylation level of P65 and IKBa, which were elevated by IL-1 $\beta$ treatment, indicating that MLT can attenuate the IL-1 $\beta$-induced activation of NF-KB signaling.

Conclusion: The current evaluation showed that MLT can save IL-1 $\beta$-impaired chondrogenesis of human BMSCs by restoring the pellet size and matrix accumulation, and maintaining the metabolic balance, reducing cell apoptosis. Our study also showed that MLT can attenuate the IL-1 $\beta$-induced activation of the NF-KB signaling pathway, which is the most important pathway downstream of $\mathrm{IL}-1 \beta$, and plays a crucial role in inflammation, apoptosis, and metabolism. Thus, MLT has prospects for treating OA due to its multifaceted functions, such as mitigating inflammation, maintaining metabolic balance, and mitigating apoptosis.

Keywords: Human mesenchymal stem cells, Chondrogenesis, Melatonin, Interleukin-1 $\beta$, Metabolic balance, Apoptosis

\section{Background}

Osteoarthritis (OA) is the most widespread degenerative arthritic disease across the globe, and it is a primary cause of disability, with radiographically determined OA impacting about $37 \%$ of the US population older than 60 years of age $[1,2]$. Typical clinical characteristics include pain, joint dysfunction, and deformity, which all lower health-related quality of life. OA is anticipated to be the fourth-leading reason for disability by the year 2020 because of the aging of the world's population [3]. This motivates us to further explore more OA treatment options, including stem-cell-based therapy.

OA has been thought of as a degenerative disease of the cartilage for a long time; however, increasing evidence suggests that inflammation plays a pivotal part in its pathogenesis. Inflammation takes part in the early course of OA, resulting in the metabolic dysfunction of chondrocytes, advancing the malfunction of articular cartilage, and eventually leads to the functional breakdown of synovial joints. Interleukin-1 $\beta$ (IL-1 $\beta$ ) is a primary mediator of local inflammatory processes in OA $[4,5]$. There are extensive studies that suggest elevated levels of IL-1 $\beta$ during the cartilage destruction cascade in the OA process $[4,6,7]$. IL- $1 \beta$ can change the differentiation and function of chondrocytes, which can then prompt the expression and activation of matrix metalloproteinases (MMPs) and a disintegrin and metalloproteinase with thrombospondin motifs (ADAMTS), enzymes that break down the cartilage matrix, encourage cell apoptosis, and are believed to be the downstream effectors of OA pathogenesis [4, 8-10]. Moreover, there is extensive literature that demonstrates the effects of IL-1 $\beta$ on chondrogenic MSCs $[5,11]$. Wehling et al. [11] found that IL-1 $\beta$ inhibited chondrogenesis of MSCs in a dose-dependent manner and cell-based repair of lesions in articular cartilage will be compromised in inflamed joints.

Contemporary treatments for OA mainly focused on pain management, viscosupplementation, and joint replacement, which all simply target the clinical symptoms of the progressive stage of OA [7]. Drugs focused on fixing the injured cartilage due to $\mathrm{OA}$ are desperately required. Possible treatments, including anti-IL-1 $\beta$, in OA animal models revealed lowered infiltration of inflammatory cells and cartilage injury [12]. Unfortunately, IL-1 $\beta$ blockade is linked to liver toxicity $[13,14]$. Since articular cartilage has a limited self-repair capacity, the use of BMSCs to regenerate cartilage is an attractive approach due to the multiple differentiation abilities and the extensive resources of harvestable BMSCs available $[15,16]$. MSCs, which inhabit bone marrow and numerous adult tissues, are able to self-renew and differentiate into various cell lineages, such as osteoblasts and chondrocytes. MSCs have been established in healthy and damaged cartilage and seem to keep at a minimum some promising ability to regenerate cartilage $[17,18]$. Cartilage tissue-engineering repair strategies that depend on the chondrogenesis of MSCs are attractive, but in instances of OA they require chondrogenesis to take place within an inflamed environment. Moreover, MSCs interact with both the innate and adaptive immune systems, generally leading to abatement of ongoing inflammatory responses, which aggravates the damage caused by inflammatory factors like IL-1 $\beta$ [19]. However, there is surprisingly little in the literature concerning ways to stop or reverse IL-1 $\beta$-induced impairment of chondrogenesis, which could greatly improve the clinical outcomes of cartilage tissue-engineering repair strategies for OA treatment $[5,11,20]$. 
Melatonin (MLT), best known as a modulator of circadian rhythms [21, 22], is reported to have multiple functions, including restriction of tumor development, immunomodulation, and antioxidation [23-27]. MLT and its metabolites modulate a variety of molecular signaling pathways including proliferation, apoptosis, metastasis, and inflammation, across a wide range of pathophysiological situations [28-30]. Further, MLT plays a pivotal part in managing skeleton establishment and growth. Our prior evaluation revealed that MLT can halt adipogenesis and encourage both osteogenic and chondrogenic differentiation of MSCs [31-33]. IL-1 $\beta$ is an important ligand of the NF- $\mathrm{kB}$ pathway, which is one of the most important pathways involved in inflammation and apoptosis. We believe that the NF- $\mathrm{kB}$ pathway plays a crucial role in IL-1 $\beta$ 's inhibitory effects in the process of chondrogenesis. With its powerful anti-inflammatory and prochondrogenic effects, we suggest that MLT could be a potential therapeutic compound for IL-1 $\beta$-inhibited chondrogenesis by suppressing the activation of NF- $\mathrm{KB}$ signaling.

In the current evaluation, MLT was examined for its potential to encourage chondrogenic differentiation, retain metabolic balance, and lower cell apoptosis of human MSCs with the inflammatory factor IL- $1 \beta$. MLT's influence on the NF- $\kappa B$ pathway was also assessed. The objectives of this evaluation are to additionally establish the main part of MLT in the management of the differentiation of MSCs in a pathological environment and its potential underlying mechanism, offering additional evidence for the utilization of MLT in stem-cell-based OA treatment.

\section{Methods}

\section{Antibodies and reagents}

Recombinant human IL-1 $\beta$ was purchased from R\&D (Minneapolis, MN, USA). MLT, Alcian blue solution, hydrochloride, EDTA, 1,9-dimethylmethylene blue (DMMB), and dye Hoechst 33,258 were purchased from Sigma-Aldrich (St. Louis, MO, USA). COL2A1 antibody was from Abcam (Cambridge, UK). The DAB Horseradish Peroxidase Color Development Kit was from (Beyotime Biotechnology, Beijing, China), and the MEBSTAIN Apoptosis TUNEL Kit Direct was from MBL International Co. (Woburn, MA, USA). The subsequent antibodies (Abs) were bought from Cell Signaling Technology (CST, Danvers, MA, USA): $\mathrm{P} 65$, phospho- $\mathrm{P} 65$, ІкB $\alpha$, phospho-ІкB $\alpha$, GAPDH, goat anti-rabbit IgG H\&L (HRP), and goat anti-mouse IgG H\&L (HRP).

\section{Separation and culture of MSCs}

The study was authorized by the Ethical Committee of Sun Yat-sen University, and written informed consent was gained from each of the participants enrolled in the evaluation. MSCs were separated from bone marrow obtained from healthy volunteer donors as described previously [31, 33]. In short, the bone marrow specimens were diluted with PBS. Cells were then fractionated on a lymphoprep density gradient by centrifugation at $500 \times g$ for $20 \mathrm{~min}$. Interfacial mononuclear cells were gathered, resuspended in low-glucose Dulbecco's modified Eagle medium (DMEM; Gibco, Waltham, MA, USA) augmented with $10 \%$ FBS (Gibco), and then seeded and incubated at $37^{\circ} \mathrm{C} / 5 \% \mathrm{CO}_{2}$. After $48 \mathrm{~h}$, nonadherent cells were eliminated by replacing the medium with fresh medium. The medium was then replaced every 3 days. When the cells approached $80-90 \%$ confluence, they were trypsinized, quantified, and plated again. Cells from passages 3-6 were utilized for the experiments.

\section{Chondrogenic differentiation}

A high-density micromass culture system was used for the chondrogenic differentiation of human MSCs as described previously [33]. In short, MSCs were trypsinized, washed, and then resuspended at $2 \times 10^{7}$ cells $/ \mathrm{ml}$ in OriCell $^{\text {tm }}$ Human Mesenchymal Stem Cell Chondrogenic Differentiation Medium (Cyagen Biosciences Inc.). Droplets $(12.5 \mu \mathrm{l})$ were carefully placed in each interior well of a 24-well plate. Cells were allowed to adhere at $37{ }^{\circ} \mathrm{C}$ for $2 \mathrm{~h}$, followed by addition of $500 \mu \mathrm{l}$ chondrogenic medium containing vehicle, $10 \mathrm{ng} / \mathrm{ml} \mathrm{IL-1} \beta$ and vehicle (PBS with $0.1 \%$ BSA), or $10 \mathrm{ng} / \mathrm{ml} \mathrm{IL-1} \beta$ and $50 \mathrm{nM}$ MLT. The medium was replaced every 3 days and the pellets were harvested on days 7, 14, and 21.

\section{Real-time RT-PCR assay}

Total RNA was removed with RNAiso Plus Reagent (Roche, Basel, Switzerland) and then changed to cDNA with PrimeScript ${ }^{\mathrm{Tw}}$ RT Master Mix (Roche) based on the manufacturer's instructions. Real-time PCR was conducted on a Light Cycler 480 Real-Time PCR Detection System (Roche) with SYBR Green I Master Mix (Roche). Expression levels were established for the following genes: ACAN, COL2A1, COL10A1, SOX9, MMP9, $M M P 13$, and ADAMTS4. The expression level of the glyceraldehyde-3-phosphate dehydrogenase (GAPDH) gene acted as a reference. Every PCR was processed in triplicate. The $\mathrm{Ct}$ value of GAPDH was subtracted from the $\mathrm{Ct}$ value of the target gene $(\Delta \mathrm{Ct})$, and the average $\Delta \mathrm{Ct}$ value of each replicate was documented. The relative expression levels of every gene were established with the $2^{-\Delta \Delta \mathrm{Ct}}$ method. Primer sequences utilized in this evaluation are presented in Table 1.

\section{Alcian blue staining}

Micromasses were fixed in 4\% paraformaldehyde for $3 \mathrm{~h}$ and then dehydrated with ethanol, washed with xylene, and embedded in paraffin. Sections with a thickness of $4 \mu \mathrm{m}$ were cut and coated on the glass slides. We then 
Table 1 All primers for teal-time RT-PCR assay

\begin{tabular}{ll}
\hline Gene & Primer sequence \\
\hline COL2A1 & Sense: 5'-GGCAATAGCAGGTTCACGTACA-3' \\
ACAN & Antisense: 5'-CGATAACAGTCTTGCCCACT-3' \\
SOX9 & Sense: 5'-TGCATTCCACGAAGCTAACCTT-3' \\
& Antisense: 5'-GACGCCTCGCCTTCTTGAA-3' \\
COL10A1 & Sense: 5'-AGCGAACGCACATCAAGAC-3' \\
& Antisense: 5'-GCTGTAGTGTGGAGGTGAA-3' \\
MMP9 & Sense: 5'-CAAGGCACCATCTCCAGGAA-3' \\
& Antisense: 5 '-AAAGGGTATTGTGGCAGCATATT-3' \\
MMP13 & Sense: 5'-TGTACCGCTATGGTACACTCG-3' \\
& Antisense: 5 '-GGCAGGGACAGTTGCTTCT-3' \\
ADAMTS4 & Sense: 5'-CCAGACTTCACGATGGCATTG-3' \\
& Antisense: 5'-GGCATCTCCTCCATAATTTGGC-3' \\
GAPDH & Sense: 5'-GAGGAGGAGATCGTGTTCCA-3' \\
& Antisense: 5 '-CCAGCTCTAGTAGCAGCGTC-3' \\
& Sense: 5'-AGAAAAACCTGCCAAATATGATGAC-3' \\
& Antisense: 5 '-TGGGTGTGCTGTTGAATC-3'
\end{tabular}

deparaffinized the slides and hydrated them three times with distilled water, and Alcian blue solution $(\mathrm{pH} 2.5$; Sigma-Aldrich) was added and incubated for $1 \mathrm{~h}$ at room temperature. After a removal of staining reagents, the slides were washed in running tap water for $2 \mathrm{~min}$. Then mount with resinous mounting medium. Finally, the sections were photographed with an Olympus BX51 microscope (Olympus, Tokyo, Japan).

\section{Quantitative analysis of glycosaminoglycan}

Pellets were cleaned and digested in PBS with $0.03 \%$ papain (Merck, Darmstadt, Germany), $5 \mathrm{mM}$ cysteine hydrochloride, and $10 \mathrm{mM}$ EDTA for $16 \mathrm{~h}$ at $65^{\circ} \mathrm{C}$. The glycosaminoglycan (GAG) concentration was quantified with a 1,9-dimethylmethylene blue dye binding assay. In short, a portion of the lysate was reacted with DMMB solution for $10 \mathrm{~min}$, and the absorbance at $525 \mathrm{~nm}$ was established with Varioskan Flash (Thermo Scientific, Waltham, MA, USA). Pellet digests were taken through three freeze-thaw cycles, and aliquots were added to $100 \mathrm{ng} / \mathrm{ml}$ of Hoechst Dye 33,258 (Sigma) in $10 \mathrm{mM}$ Tris ( $\mathrm{pH}$ 7.4), $1 \mathrm{mM}$ disodium EDTA, and $100 \mathrm{mM} \mathrm{NaCl}$. DNA concentration was determined by fluorescent dye Hoechst 33,258 binding assay with a SpectraMax M5 microplate reader (Molecular Devices, Sunnyvale, CA, USA). Fluorescence was measured using excitation and emission wavelengths of $485 \mathrm{~nm}$ and $528 \mathrm{~nm}$, respectively, and DNA concentrations were determined relative to a lambda DNA standard curve. For GAG synthetic activity, the resulting GAG amounts were normalized to the amount of DNA for each sample.

\section{Immunohistochemical analysis}

We used 4\% paraformaldehyde for fixation of the tissues at room temperature for $1 \mathrm{~h}$. Paraffin sections $(4 \mu \mathrm{m}$ thick) were prepared and immunohistochemical (IHC) analysis was performed using a Histostain-Plus Kit (Thermo Fisher Scientific, Waltham, MA, USA). We used $5 \%$ bovine serum albumin as the blocking reagent. The specimen was treated for $30 \mathrm{~min}$ at room temperature, and the tissues were incubated with the anti-COL2A1 antibody (1:500) at $4{ }^{\circ} \mathrm{C}$ overnight. Detection was performed with a DAB Horseradish Peroxidase Color Development Kit (Origin Technologies, Inc.).

\section{Terminal deoxynucleotidyl-transferase-mediated dUTP nick end labeling assay}

A terminal deoxynucleotidyl-transferase-mediated dUTP nick end labeling (TUNEL) assay was conducted with a MEBSTAIN Apoptosis TUNEL kit direct (MBL International Co.) based on the manufacturer's instructions. The percentage of TUNEL-positive cells relative to propidium iodide (PI)-stained cells was calculated. Three independent experiments were conducted and quantified for each experimental group.

\section{Immunoblotting analysis}

Pellets were cleaned three times with cold PBS and gathered in RIPA (Beyotime, Shanghai, China), adding 1\% protease inhibitor and phosphatase inhibitor. Pellets were exposed to the liquid nitrogen for $15 \mathrm{~min}$ and pellet lysates were obtained using a TissueLyser (QIAGEN, Germany). Identical portions of each specimen were subjected to SDS-polyacrylamide gel electrophoresis (PAGE) and moved to PVDF transfer membranes (Millipore). Membranes were halted with $5 \%$ nonfat milk for $1 \mathrm{~h}$ at room temperature and then incubated with the anti-P65, anti-phospho-P65, anti-IK $\mathrm{B} \alpha$, and anti-phospho-IкB $\alpha$ specified antibodies (1:1000) at $4{ }^{\circ} \mathrm{C}$ overnight. Antibody-specific labeling was noted by incubation with secondary antibodies (1:2000) for $1 \mathrm{~h}$ at room temperature and observed with an ECL kit (Millipore). The band was established with ImageJ software and normalized to GAPDH (1:2000) as the loading control.

\section{Statistical analysis}

Comparisons of perimeters, GAG content, gene expressions, and quantitation of protein expressions were performed using a two-tailed independent Student's $t$ test. Statistical analyses for comparisons of apoptosis rate and relative protein expressions were performed using chi-square or Fisher's exact tests when appropriate. All statistical analyses were conducted with SPSS 20.0 statistical software (SPSS, Chicago, IL, USA) and GraphPad Prism 5.01. The level of statistical significance was established at $P<0.05$.

\section{Results}

To examine the impacts of MLT on chondrogenic differentiation of MSCs with IL-1 $\beta$, chondrogenic differentiation was prompted in human MSCs in chondrogenic 
medium with vehicle, $10 \mathrm{ng} / \mathrm{ml} \mathrm{IL-1 \beta}$ and vehicle, or $10 \mathrm{ng} / \mathrm{ml} \mathrm{IL-1} \beta$ and $50 \mathrm{nM}$ MLT. No difference was discovered between groups in pellet size on day 7 of chondrogenesis (Fig. 1a); quantitative analysis of the perimeters of the pellets also confirmed that there was no difference in chondrogenesis on day 7 between different groups (Fig. 1b). However, the cartilage pellets treated with IL-1 $\beta$ were smaller than those of the controls in the 14-day and 21-day groups and additional MLT treatment partially restored the size of the pellets (Fig. 1cf-f).

Alcian blue staining was used for evaluation of cartilage matrix synthesis and accumulation. The results of
Alcian blue staining and the quantitative analysis of glycosaminoglycan (GAG) showed that GAG synthesis and matrix deposition decreased in the presence of IL- $1 \beta$ and was elevated by MLT treatment on day 7 (Fig. 2a, b), day 14 (Fig. 2c, d), and day 21 (Fig. 2e, f) $(P<0.05)$. These outcomes revealed that IL-1 $\beta$ suppresses the accumulation of matrix during chondrogenesis of MSCs, and MLT can rescue the impacts of IL-1 $\beta$.

To further confirm the effects of IL-1 $\beta$ and MLT on the process of chondrogenesis, the level of expression of the typical chondrogenic marker COL2A1 was detected using real-time RT-PCR and immunohistochemical (IHC) staining. These findings revealed that IL-1 $\beta$

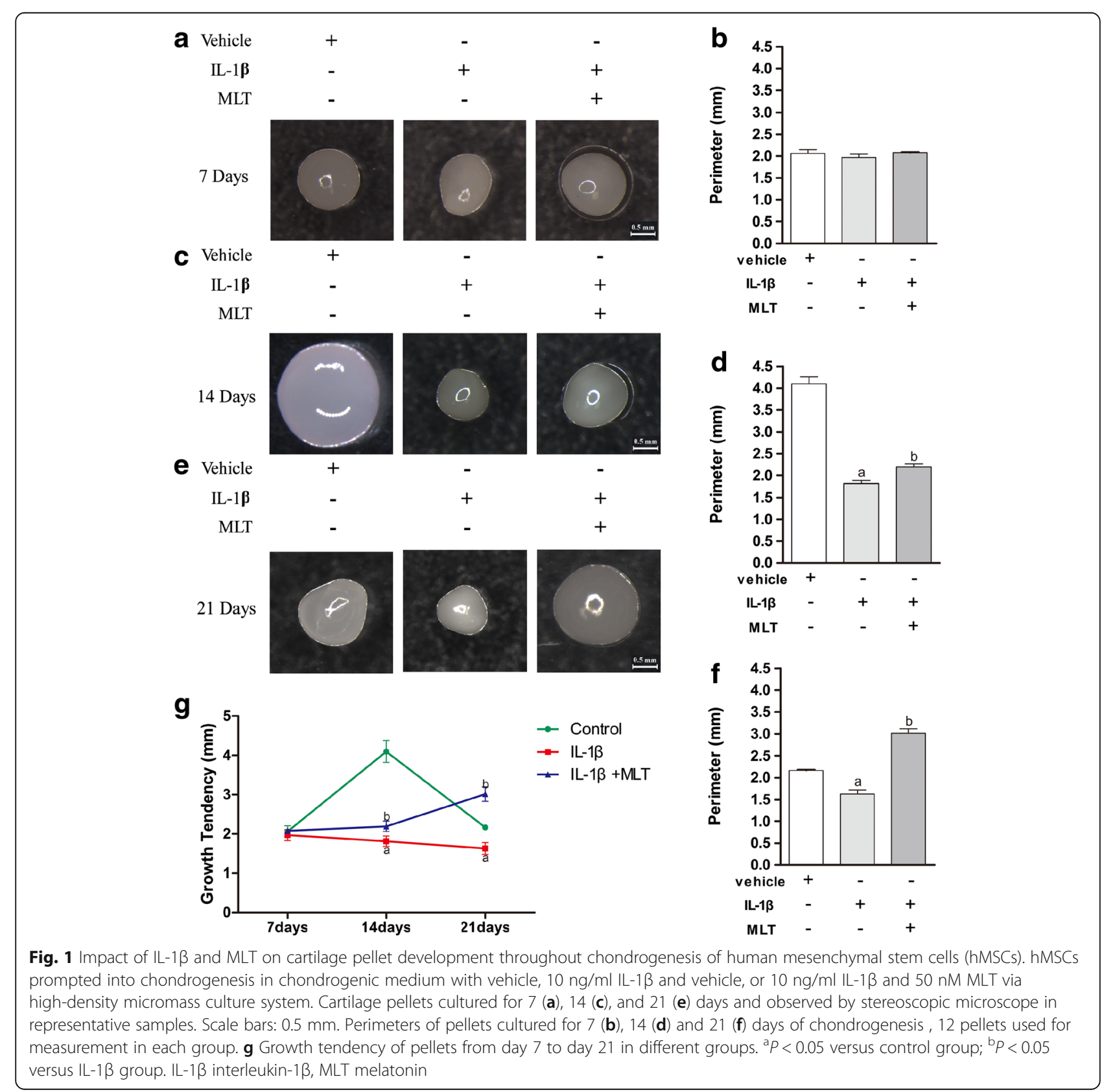




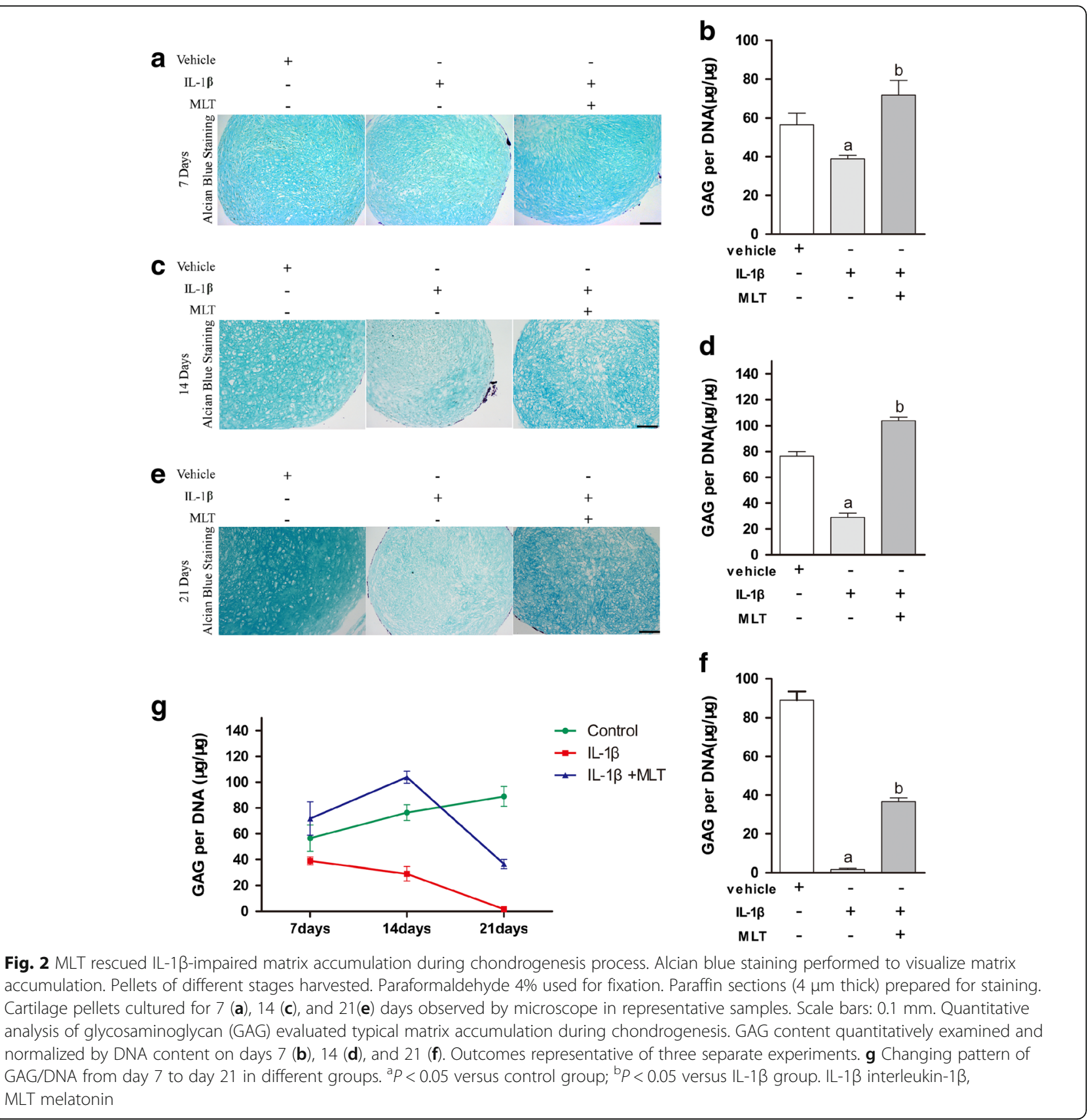

dramatically inhibited collagen II expression on day 7 (Fig. 3a, b), day 14 (Fig. 3c, d), and day 21 (Fig. 3e, f) during chondrogenesis, while the additional MLT treatment reversed this situation at both mRNA and protein levels $(P<0.05)$. These results showed that IL-1 $\beta$ suppresses gene and protein expression of typical chondrogenic marker COL2A1 of chondrogenic MSCs. Again, MLT can counterbalance the effects of IL-1 $\beta$.

Next, RT-PCR was utilized to explore the impacts of IL-1 $\beta$ and MLT on the expressions of other chondrogenic markers, such as ACAN, COL1OA1, and SOX9. As shown in Fig. 4a, b, the level of expression of $A C A N$ and
SOX9 are consistent with the pattern of expression of COL2A1. IL-1 $\beta$ downregulated ACAN and SOX9 expression, whereas after the addition of MLT to IL-1 $\beta$, upregulation of those genes was were observed on days 7 and $14(P<0.05)$. Of note, on day 21 the effects of IL-1 $\beta$ and MLT on $A C A N$ were consistent with those on days 7 and $14 \quad(P<0.05)$, while the effect of MLT on SOX9 was gone. COL1OA1, a chondrogenic and a hypertrophic marker, was found to be elevated by IL-1 $\beta$ treatment and then declined after the addition of MLT on day 7 (Fig. 4c) $(P<0.05)$. IL-1 $\beta$ downregulated COL10A1 expression, whereas MLT reversed the 

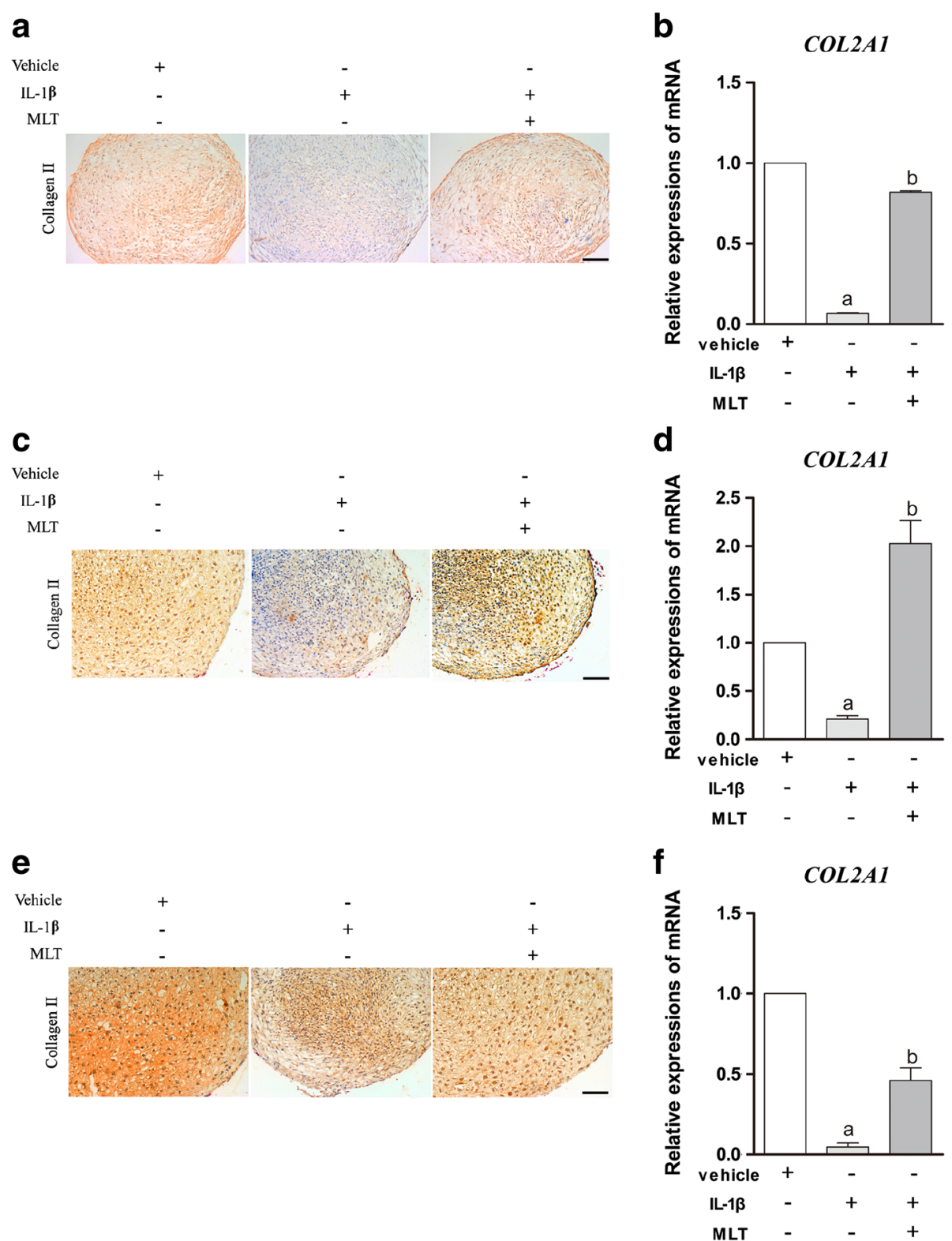

Fig. 3 MLT reversed IL-1 $\beta$-inhibited COL2A1 expression at both mRNA and protein levels. QPCR and IHC analysis assessed COL2A1 expressions at 7, 14 and 21 days. mRNA expression of COL2A1 measured by qPCR and normalized to GAPDH. Relative expression levels of gene on 7 (b), 14 (d), and 21 (f) days representative of three independent experiments. ${ }^{a} P<0.05$ versus control group; ${ }^{b} P<0.05$ versus IL-1 $\beta$ group. IHC staining of collagen type II performed after 7 (a), 14 (c), and 21 (e) days of differentiation. Scale bars: 0.1 mm. IL-1 $\beta$ interleukin-1 $\beta$, MLT melatonin

effect on day 14. The effect of MLT on COL10A1 was also gone on day 21 . We also explored the impacts of IL-1 $\beta$ and MLT on catabolic and proapoptotic markers like MMP9, MMP13, and ADAMTS4. On day 7, IL-1 $\beta$ treatment had little effect on MMP9 expression, while MLT treatment decreased the expression of MMP9; IL-1 $\beta$ then upregulated MMP9 expression on days 14 and 21, while MLT downregulated the level of expression of MMP9 (Fig. $4 \mathrm{~d})(P<$ $0.05)$. IL- $1 \beta$ upregulated MMP13 and ADAMTS4 expression, whereas the addition of MLT to IL-1 $\beta$ then downregulated their expression on days 7,14 , and 21 (Fig. 4e, f) $(P<0.05)$. The impacts of IL-1 $\beta$ and MLT on these proapoptotic markers suggested that MLT could have a part in the chondrogenesis process as an anticatabolic and antiapoptotic agent.

We performed a TUNEL assay to determine how IL- $1 \beta$ and MLT influenced the cell fate of chondrogenic MSCs. IL-1 $\beta$ treatment increased the percentage of TUNEL-positive cells compared to control, and the addition of MLT significantly reversed this effect mainly on day 7 (Fig. 5a, b), day 14 (Fig. 5c, d), and day 21 

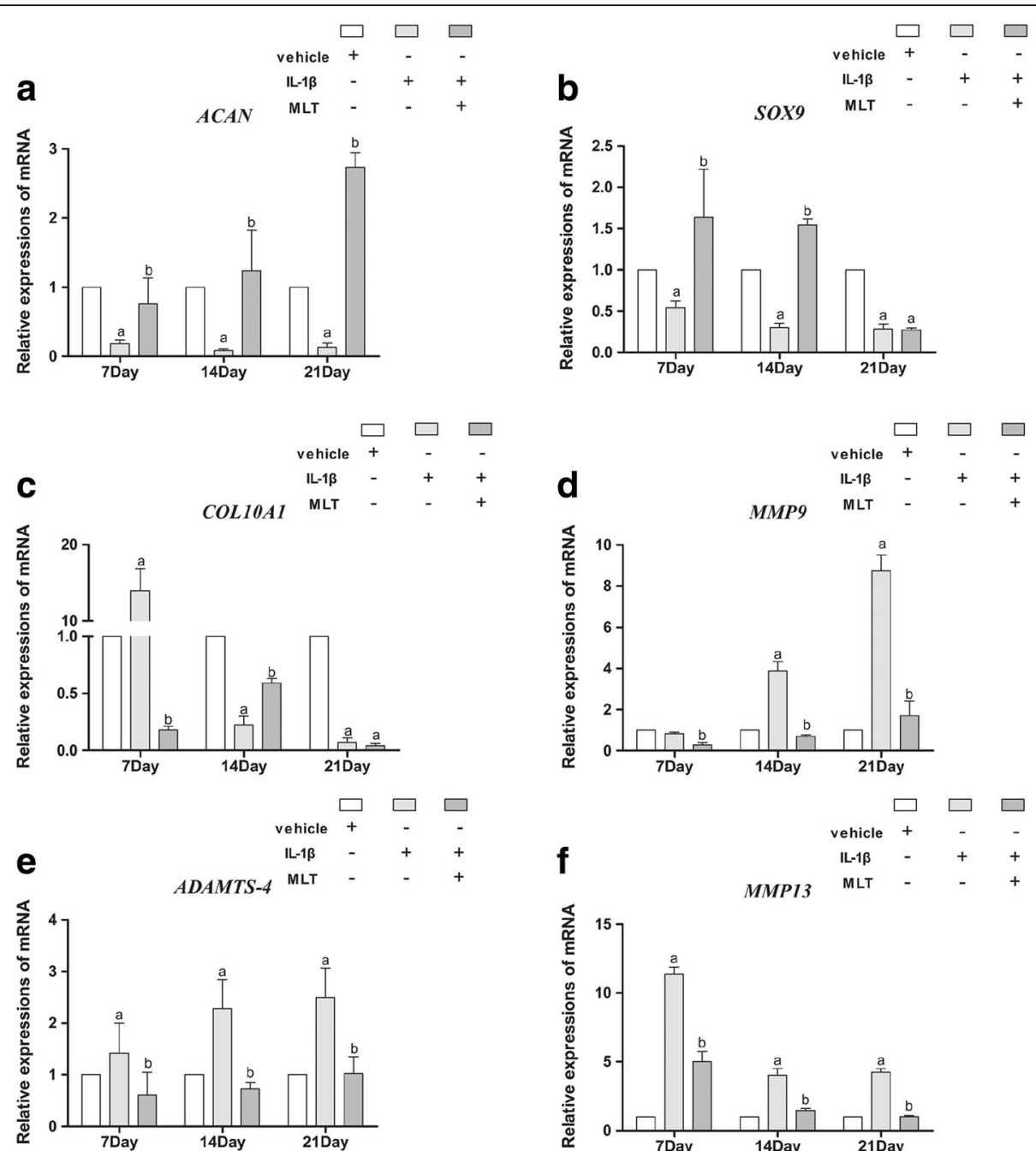

Fig. $4 \mathrm{IL}-1 \beta$ and MLT treatment affected chondrogenic and apoptotic marker expression at mRNA level. Expression of ACAN (a), SOX9 (b), COL1OA1 (c), MMP9 (d), MMP13 (e), and ADAMTS4 (f) measured by qPCR and normalized to GAPDH. Relative expression level of each gene on different stages of differentiation representative of three independent experiments. ${ }^{\mathrm{a}} P<0.05$ versus control group; ${ }^{b} P<0.05$ versus IL-1 $\beta$ group. IL-1 $\beta$ interleukin-1 $\beta$, MLT melatonin

(Fig. 5e, f) $(P<0.05)$. These results confirmed that IL-1 $\beta$ induces MSC apoptosis during the process of chondrogenesis and MLT plays the role of an antiapoptotic agent, reducing MSC apoptosis and rescuing IL-1 $\beta$-impaired chondrogenesis.

The NF- $\mathrm{kB}$ pathway is one of the most crucial pathways in apoptosis, and IL- $1 \beta$ is a pivotal ligand of the NF-KB pathway. We believe that the NF- $\kappa B$ pathway plays a crucial part in IL-1ß's inhibitory effects in chondrogenesis. To test this hypothesis, we determined the levels of expression and activity of key molecules of the NF-kB pathway of the pellets on day 21 using immunoblotting. As shown in Fig. $6 a$, both IL-1 $\beta$ and MLT regulated the phosphorylation levels of p-P65 and p-IкB $\alpha$. While the expression of total P65 was downregulated by IL-1 $\beta$, MLT then elevated the P65 level $(P<0.05)$. Total IкB $\alpha$ remained the same in different groups (Fig. 6b); however, IL-1 $\beta$ upregulated the phosphorylation levels of P65 and IKB $\alpha$, causing increased NF- $\mathrm{kB}$ activation. In contrast, MLT downregulated the phosphorylation levels of $\mathrm{P} 65$ and IкB $\alpha$, thus attenuating NF- $\mathrm{kB}$ activation (Fig. $6 \mathrm{c}, \mathrm{d})(P<0.05)$. These results indicated that MLT attenuated IL-1 $\beta$ 's impact on the NF- $k B$ signaling pathway.

\section{Discussion}

MSCs have already been intensively examined and utilized in clinical trials for regenerative therapies in the skeletal system [17, 34]. Recent studies demonstrate that MSCs may act as anti-inflammatory agent to allow the joint to self-repair; MSCs combined with appropriate scaffolds can form cartilaginous or even osseous compartments to repair cartilage [35]. However, such 
a

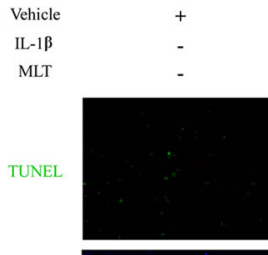

DAP I
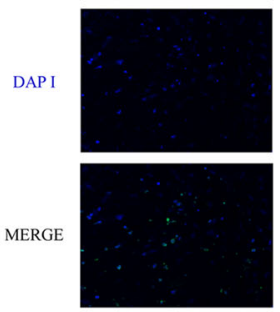

Cehicle
IL-1
MLT

-
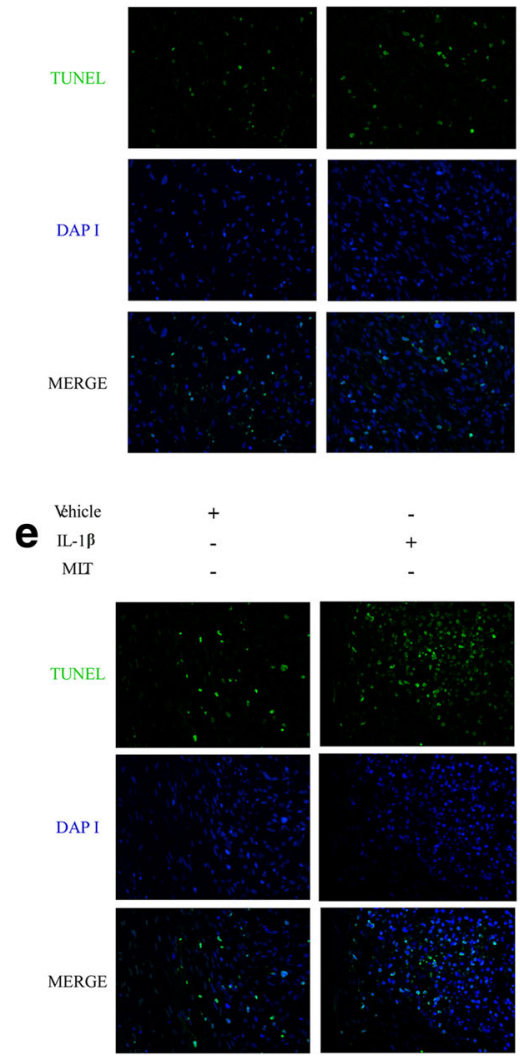
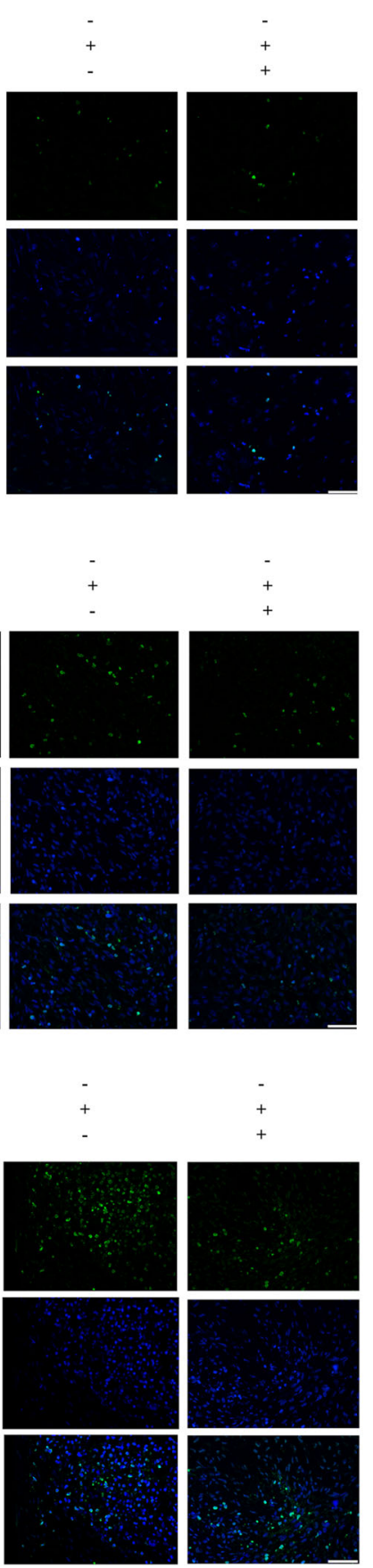

b

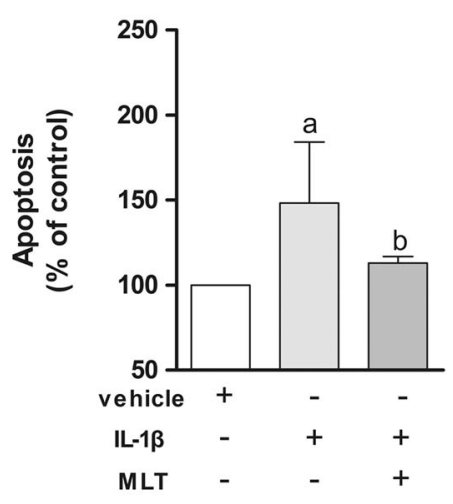

d

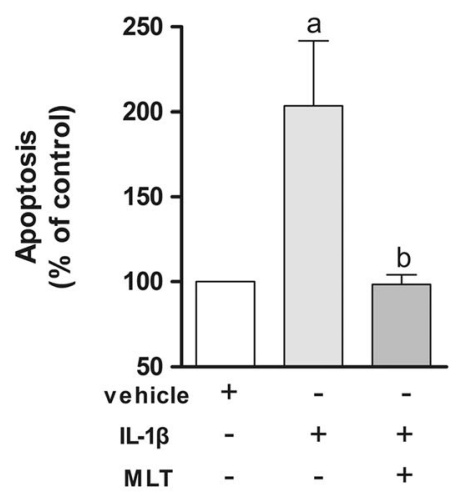

f

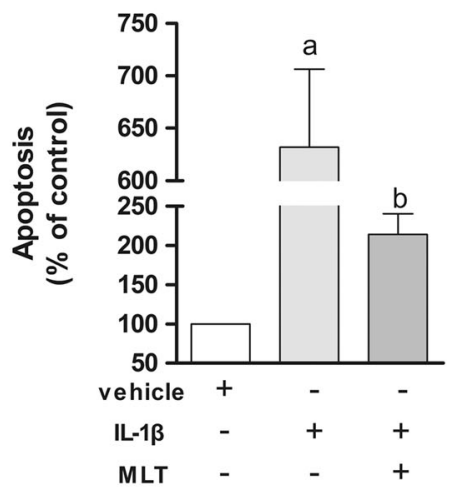

Fig. 5 MLT protected human MSCs from IL-1ß-induced apoptosis in process of chondrogenesis. TUNEL assay showed apoptotic MSCS (green fluorescence) with Pl-labeled nuclei (blue fluorescence) on days 7 (a), 14 (c), and 21 (e). Positive rates statistically analyzed on right correspondingly $(\mathbf{b}, \mathbf{d}, \mathbf{f})$, and data represent mean \pm SD of three independent experiments. Scale bars $=50 \mu \mathrm{m}$. ${ }^{\mathrm{a}} P<0.05$ versus control group; ${ }^{\mathrm{b}} P<0.05$ versus IL-1 $\beta$ group. DAPI 4',6-diamidino-2-phenylindole, IL-1 $\beta$ interleukin-1 $\beta$, MLT melatonin, TUNEL terminal deoxynucleotidyl-transferase-mediated dUTP nick end labeling

therapies have not been successful thus far and they did not have the intended impact [34]. A large issue that researchers encounter is the inflamed surroundings $[5,7,11]$. Our intention is to use the in-vitro chondrogenesis model to test the possible effect of
MLT on IL-1 $\beta$-impaired cartilage formation and the underlying mechanism. We want to prove that MLT can act as a possible drug that helps to optimize the cartilage tissue engineering system or even a drug that helps the cartilage to self-repair under 


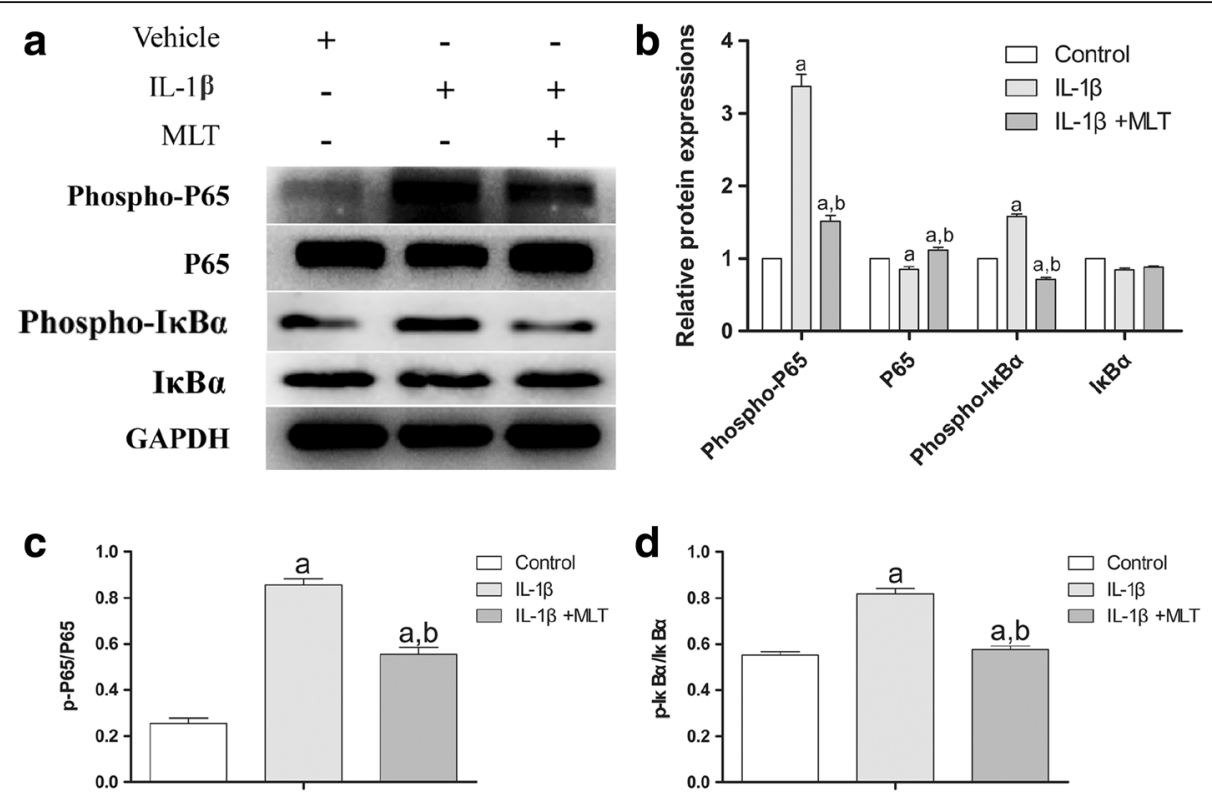

Fig. 6 MLT downregulated IL-1 $\beta$-enhanced phosphorylation of P65 and IKBa in NF-KB pathway. Immunoblotting (a) conducted to detect expression and total and phosphorylated P65 and IKBa to reflect activation of NF-KB signaling. Phospho-P65, P65, phospho-IKBa, and IkBa protein levels established (b). Ratio of relative protein expression of p-P65 to relative protein expression of P65 (p-P65/P65) (c), and ratio of relative protein expression of $\mathrm{p}-\mathrm{I} \mathrm{KBa}$ to relative protein expression of $\mathrm{IKBa}(\mathrm{p}-\mathrm{IKBa} / \mathrm{I} \mathrm{KBa})$ examined $(\mathbf{d}) .{ }^{\mathrm{a}} \mathrm{P}<0.05$ versus control group; ${ }^{\mathrm{b}} P<0.05$ versus $\mathrm{IL}-1 \beta$ group. IL-1 $\beta$ interleukin-1 $\beta$, MLT melatonin

inflammatory conditions. To the best of our knowledge, our evaluation is the first to discover that MLT can function as a proanabolic, anticatabolic, and antiapoptotic agent for MSCs in the chondrogenic process under IL-1 $\beta$ challenge.

The majority of previous studies $[5,11]$ that assessed either the effect of inflammatory cytokines or MLT have been based on a single point in time, which disregards valuable information in the whole process of chondrogenesis. Liu et al. [5] investigated the role of MLT on proinflammatory cytokine-inhibited chondrogenesis in synovium mesenchymal stem cells and the possible role of reactive oxygen species in its process. They found that the chondroprotective effect of MLT was potentially correlated to decreased ROS, preserved SOD, and suppressed levels of MMPs. However, in the current study, we used BMSCs induced for 7, 14, and 21 days to help monitor the chondrogenic process in a continuous way, through which we found that on day 7 IL- $1 \beta$ and MLT barely affected the chondrogenesis in pellet size and matrix accumulation. Instead, on day 21 the most obvious effect of IL-1 $\beta$ and MLT was observed among the different stages. Specifically, on day 7 the induced cartilage pellets showed no difference in size or perimeter or Alcian blue staining among different groups, while the GAG content, COL2A1, ACAN, and SOX9 expression was altered by IL- $1 \beta$ and MLT treatment. Of note, the mRNA level of COL10A1, which is a cartilage hypertrophic marker, was surprisingly upregulated in the
IL-1 $\beta$ group. Surprisingly, MLT downregulated the expression level of COL1OA1. Since hypertrophy is the terminal stage of cartilage before apoptosis, this result would lead us to realize that apoptosis of induced MSCs may be partially responsible for unsatisfactory outcomes of chondrogenesis in an inflamed environment. On days 14 and 21 , pellets treated with IL-1 $\beta$ showed smaller size and perimeter, decreased matrix accumulation, and downregulation of typical chondrogenic marker COL2A1 at both mRNA and protein levels, whereas MLT restored the effects. $A C A N$ shared the same changing patterns with COL2A1 only on day 14 . SOX9, a marker of cartilage formation, remained at low levels in the IL- $1 \beta$ and MLT groups, suggesting 21 days is a terminal stage of chondrogenesis in the presence of IL-1 $\beta$. However, MMP9, MMP13, and ADAMTS4, known as catabolic and proapoptotic markers, were upregulated across all three stages in the IL-1 $\beta$ group, and addition of MLT reversed the expression levels of the three genes. Lastly, the TUNEL assay confirmed the hypothesis that MLT plays a role in rescuing IL-1 $\beta$-impaired chondrogenesis as an antiapoptotic agent.

Our study has some limitations. Firstly, IL- $1 \beta$ is a highly crucial inflammatory factor, and it has a pivotal part in the pathogenesis of OA. Apart from IL-1 $\beta$, numerous soluble inflammatory mediators (TNF- $\alpha$, IL-6, etc.) have been determined to be present in OA joint tissues and fluids [7, 36]. However, Liu et al. [5] demonstrated that IL- $1 \beta$ and TNF- $\alpha$ had an inhibitory impact 
on the chondrogenesis of MSCs. IL-1 $\beta$ was found to have a more potent effect than TNF- $\alpha$. Thus, we chose IL-1 $\beta$ as representative to create an inflamed environment for MSCs. Secondly, our study lacks evidence from the protein level. MMPs and collagens are important proteins evolving in the cartilage metabolism. We use $M M P 9 / M M P 13 / C O L 10 A 1$ gene expression as catabolic, proapoptotic, and hypertrophic markers. We will continue our work to explore the protein expression and functions of MMPs and ColX. Thirdly, we did not fully excavate the underlying mechanisms of MLT's effect. Guo et al. [37] demonstrate that MLT inhibits Sirt1-dependent NAMPT and NFAT5 signaling in chondrocytes to attenuate OA. However, in the chondrogenic process with IL- $1 \beta$ presence, MLT's role is still not clear. Apoptosis signaling pathways in $\mathrm{OA}$ and the potential protective part of MLT are well established [38]. The NF- $\mathrm{kB}$ signaling pathway is crucial in the apoptosis of chondrocytes. IL- $1 \beta$ is a classical ligand of the NF- $\kappa B$ pathway. To test this hypothesis, we detected the expression level and activity of key molecules of the NF- $\mathrm{kB}$ pathway. The results showed that on day 21 , IL- $1 \beta$ upregulated the phosphorylation levels but not the expression of $\mathrm{P} 65$ and $\mathrm{I} \kappa \mathrm{B} \alpha$, causing increased NF- $\mathrm{KB}$ activation. In contrast, MLT downregulated the phosphorylation of $\mathrm{P} 65$ and $\mathrm{I} \kappa \mathrm{B} \alpha$, thus attenuating NF- $\kappa \mathrm{B}$ signaling activation. These outcomes suggested that MLT diminished IL-1 $\beta$-induced stimulation of the NF- $\mathrm{kB}$ signaling pathway. Moreover, further study is needed to explore the exact mechanism of how MLT regulates NF- $\mathrm{KB}$ and also the possible mechanisms that involve different pathways in the process. Furthermore, the growth tendency of chondrogenic pellets in the control group shrank from day 14 to day 21 , which is not consistent with published results. We speculated that this was due to different cell sources and cell types and different methods to induce chondrogenesis. Yang et al. [39] used human adipose-derived stem cells and a pellet culture system to induce chondrogenesis, their results showing that the pellets in the control groups were largest on day 7, and shrank from day 7 to days 14 and 21 . We found in our own results that the pellets on day 14 had the most hyaline cartilage characteristics; on day 21 the pellets showed a hypertrophic cartilage phenotype, which may explain the reason why the pellets shrank. Lastly, our results were based on in-vitro data only, which limited the evidence level. We are constructing an OA rat model to testify the role of MLT, the results (data not shown) showing that MLT treatment can repair the cartilage damage caused by OA.

\section{Conclusion}

The current evaluation offers evidence that further MLT treatment can save the IL-1 $\beta$-impaired chondrogenesis of MSCs in various ways including pellet size, glycosaminoglycan accumulation, COL2A1 expression at mRNA and protein levels, and ACAN, SOX9, and COL1OA1 expression levels. Moreover, MLT may achieve this through rescuing the increased apoptosis of IL- $1 \beta$-treated MSCs and the elevated expression of $M M P 9, M M P 13$, and ADAMTS4 in the differentiating process. MLT may have rescued IL-1 $\beta$-impaired chondrogenesis of MSCs by affecting the NF- $\mathrm{kB}$ pathway. Additional evaluations have focused on unraveling the particular mechanisms through which MLT diminished IL-1 $\beta$-prompted apoptosis in MSCs and verified the therapeutic value of MLT in stem-cell-based therapies for OA.

\section{Funding}

This research was supported by the National Natural Science Foundation of China (Nos. 81572134, 81601898), the Natural Science Foundation of Guangdong Province (No. 2017A030311008), Guangzhou Science and Technology Program key projects (No. 201704020120), Natural Science Basic Research Plan in Shaanxi Province (Nos. 2017JQ8056, 2017JM8115), the China Postdoctoral Science Foundation (No. 2017M613177), and the Postdoctoral Science Foundation in Shaanxi Province of China (No. 2017BSHQYXMZZ15).

\section{Availability of data and materials}

Data sharing is not applicable to this article as no datasets were generated or analyzed during the current study.

\section{Authors' contributions}

DSH and PQS designed the experiments. BG, WJG, ZZW, TFZ, XJQ, XDW, CJL, $Y P, A J L, J C Q, Y X Z, C X X$, and $Y B L$ conducted the experiments. BG, WJG, and ZZW acquired the data. BG, WJG, DSH, and PQS analyzed the data. BG, DSH, and PQS organized the manuscript. All authors read and approved the final manuscript.

Ethics approval and consent to participate

This study was approved by the ethics committee of Sun Yat-sen Memorial Hospital, Sun Yat-sen University, Guangzhou, China.

Consent for publication

All authors approved the publication of this manuscript.

Competing interests

The authors declare that they have no competing interests.

\section{Publisher's Note}

Springer Nature remains neutral with regard to jurisdictional claims in published maps and institutional affiliations.

\section{Author details}

${ }^{1}$ Department of Orthopedics, Sun Yat-sen Memorial Hospital of Sun Yat-sen University, \#107 West Yan Jiang Road, Guangzhou 510120, Guangdong, China. ${ }^{2}$ Department of Orthopedics, The First Affiliated Hospital of Sun Yat-sen University, Guangzhou, Guangdong, China. ${ }^{3}$ Research Centre for Translational Medicine, The First Affiliated Hospital of Sun Yat-sen University, Guangzhou, Guangdong, China. ${ }^{4}$ Guangdong Provincial Key Laboratory of Orthopedics and Traumatology, The First Affiliated Hospital of Sun Yat-sen University, \#58 Zhongshan Road II, Guangzhou 510080, Guangdong, China. ${ }^{5}$ Department of Spine Surgery, Xi'an Honghui Hospital, Xi'an Jiaotong University, Xi'an, China.

Received: 30 January 2018 Revised: 15 April 2018 Accepted: 2 May 2018 Published online: 14 June 2018

References

1. Helmick CG, Felson DT, Lawrence RC, Gabriel S, Hirsch R, Kwoh CK, Liang $\mathrm{MH}$, Kremers HM, Mayes MD, Merkel PA, et al. Estimates of the prevalence of arthritis and other rheumatic conditions in the United States. Part I. Arthritis Rheum. 2008;58(1):15-25. 
2. Lawrence RC, Felson DT, Helmick CG, Arnold LM, Choi H, Deyo RA, Gabriel $\mathrm{S}$, Hirsch R, Hochberg MC, Hunder GG, et al. Estimates of the prevalence of arthritis and other rheumatic conditions in the United States. Part II. Arthritis Rheum. 2008:58(1):26-35.

3. Woolf AD, Pfleger B. Burden of major musculoskeletal conditions. Bull World Health Organ. 2003;81(9):646-56.

4. Liu-Bryan R, Terkeltaub R. Emerging regulators of the inflammatory process in osteoarthritis. Nat Rev Rheumatol. 2015;11(1):35-44.

5. Liu X, Xu Y, Chen S, Tan Z, Xiong K, Li Y, Ye Y, Luo ZP, He F, Gong Y. Rescue of proinflammatory cytokine-inhibited chondrogenesis by the antiarthritic effect of melatonin in synovium mesenchymal stem cells via suppression of reactive oxygen species and matrix metalloproteinases. Free Radic Biol Med. 2014:68:234-46.

6. Kubota E, Imamura H, Kubota T, Shibata T, Murakami K. Interleukin 1 beta and stromelysin (MMP3) activity of synovial fluid as possible markers of osteoarthritis in the temporomandibular joint. J Oral Maxillofac Surg. 1997; 55(1):20-7. discussion 27-28

7. Robinson WH, Lepus CM, Wang Q, Raghu H, Mao R, Lindstrom TM, Sokolove J. Low-grade inflammation as a key mediator of the pathogenesis of osteoarthritis. Nat Rev Rheumatol. 2016;12(10):580-92.

8. Goldring SR, Goldring MB. The role of cytokines in cartilage matrix degeneration in osteoarthritis. Clin Orthop Relat Res. 2004(427 Suppl):S27-S36.

9. Goldring MB, Goldring SR. Osteoarthritis. J Cell Physiol. 2007;213(3):626-34.

10. Chevalier X, Eymard F, Richette P. Biologic agents in osteoarthritis: hopes and disappointments. Nat Rev Rheumatol. 2013;9(7):400-10.

11. Wehling N, Palmer GD, Pilapil C, Liu F, Wells JW, Muller PE, Evans CH, Porter RM. Interleukin-1 $\beta$ and tumor necrosis factor alpha inhibit chondrogenesis by human mesenchymal stem cells through NF-KB-dependent pathways. Arthritis Rheum. 2009;60(3):801-12.

12. Joosten LA, Helsen MM, van de Loo FA, van den Berg WB. Anticytokine treatment of established type II collagen-induced arthritis in DBA/1 mice: a comparative study using anti-TNFa, anti-IL-1 $\alpha / \beta$ and IL-1Ra. Arthritis Rheum. 2008;58(2 Suppl):S110-22.

13. Cohen SB, Proudman S, Kivitz AJ, Burch FX, Donohue JP, Burstein D, Sun YN, Banfield C, Vincent MS, Ni L, et al. A randomized, double-blind study of AMG 108 (a fully human monoclonal antibody to IL-1R1) in patients with osteoarthritis of the knee. Arthritis research \& therapy. 2011;13(4):R125.

14. Chevalier X, Goupille P, Beaulieu AD, Burch FX, Bensen WG, Conrozier T, Loeuille D, Kivitz AJ, Silver D, Appleton BE. Intraarticular injection of anakinra in osteoarthritis of the knee: a multicenter, randomized, double-blind, placebo-controlled study. Arthritis Rheum. 2009;61(3):344-52.

15. Charbord P. Bone marrow mesenchymal stem cells: historical overview and concepts. Hum Gene Ther. 2010;21(9):1045-56.

16. Pittenger MF, Mackay AM, Beck SC, Jaiswal RK, Douglas R, Mosca JD, Moorman MA, Simonetti DW, Craig S, Marshak DR. Multilineage potential of adult human mesenchymal stem cells. Science (New York, NY). 1999; 284(5411):143-7.

17. Grogan SP, Miyaki S, Asahara H, D'Lima DD, Lotz MK. Mesenchymal progenitor cell markers in human articular cartilage: normal distribution and changes in osteoarthritis. Arthritis research \& therapy. 2009;11(3):R85.

18. Koelling S, Kruegel J, Irmer M, Path JR, Sadowski B, Miro X, Miosge N. Migratory chondrogenic progenitor cells from repair tissue during the later stages of human osteoarthritis. Cell Stem Cell. 2009:4(4):324-35.

19. Chow L, Johnson V, Coy J, Regan D, Dow S. Mechanisms of immune suppression utilized by canine adipose and bone marrow-derived mesenchymal stem cells. Stem Cells Dev. 2017;26(5):374-89.

20. Rainbow $R$, Ren $W$, Zeng L. Inflammation and joint tissue interactions in $O A$ implications for potential therapeutic approaches. Arthritis. 2012;2012: 741582.

21. Vriend J, Reiter RJ. Melatonin feedback on clock genes: a theory involving the proteasome. J Pineal Res. 2015;58(1):1-11.

22. Hardeland R, Madrid JA, Tan DX, Reiter RJ. Melatonin, the circadian multioscillator system and health: the need for detailed analyses of peripheral melatonin signaling. J Pineal Res. 2012;52(2):139-66.

23. Majidinia M, Sadeghpour A, Mehrzadi S, Reiter RJ, Khatami N, Yousefi B. Melatonin: A pleiotropic molecule that modulates DNA damage response and repair pathways. J Pineal Res. 2017;63:e12416. https://doi.org/10.1111/ jpi.12416.

24. Yu S, Wang X, Geng P, et al. Melatonin regulates PARP1 to control the senescence-associated secretory phenotype (SASP) in human fetal lung fibroblast cells. J Pineal Res. 2017;63:e12405. https://doi.org/10.1111/jpi.12405.
25. Li Z, Nickkholgh A, Yi X, Bruns H, Gross ML, Hoffmann K, Mohr E, Zorn M, Buchler MW, Schemmer P. Melatonin protects kidney grafts from ischemia/ reperfusion injury through inhibition of NF-kB and apoptosis after experimental kidney transplantation. J Pineal Res. 2009;46(4):365-72.

26. Regodon S, del Prado MM, Jardin I, Lopez JJ, Ramos A, Paredes SD, Rosado JA. Melatonin, as an adjuvant-like agent, enhances platelet responsiveness. J Pineal Res. 2009;46(3):275-85.

27. Weishaupt JH, Bartels C, Polking E, Dietrich J, Rohde G, Poeggeler B, Mertens N, Sperling S, Bohn M, Huther G, et al. Reduced oxidative damage in ALS by high-dose enteral melatonin treatment. J Pineal Res. 2006;41(4):313-23.

28. Lee SJ, Jung YH, Oh SY, Yun SP, Han HJ. Melatonin enhances the human mesenchymal stem cells motility via melatonin receptor 2 coupling with Galphaq in skin wound healing. J Pineal Res. 2014;57(4):393-407.

29. Wei JY, Li WM, Zhou LL, Lu QN, He W. Melatonin induces apoptosis of colorectal cancer cells through HDAC4 nuclear import mediated by CaMKII inactivation. J Pineal Res. 2015;58(4):429-38.

30. Borin TF, Arbab AS, Gelaleti GB, Ferreira LC, Moschetta MG, Jardim-Perassi BV, Iskander AS, Varma NR, Shankar A, Coimbra VB, et al. Melatonin decreases breast cancer metastasis by modulating Rho-associated kinase protein-1 expression. J Pineal Res. 2016;60(1):3-15.

31. Zhang L, Su P, Xu C, Chen C, Liang A, Du K, Peng Y, Huang D. Melatonin inhibits adipogenesis and enhances osteogenesis of human mesenchyma stem cells by suppressing PPARgamma expression and enhancing Runx2 expression. J Pineal Res. 2010:49(4):364-72.

32. Zhang L, Zhang J, Ling Y, Chen C, Liang A, Peng Y, Chang H, Su P, Huang D. Sustained release of melatonin from poly (lactic-co-glycolic acid) (PLGA) microspheres to induce osteogenesis of human mesenchymal stem cells in vitro. J Pineal Res. 2013:54(1):24-32.

33. Gao W, Lin M, Liang A, Zhang L, Chen C, Liang G, Xu C, Peng Y, Chen C, Huang $D$, et al. Melatonin enhances chondrogenic differentiation of human mesenchymal stem cells. J Pineal Res. 2014;56(1):62-70.

34. Steinert AF, Rackwitz L, Gilbert F, Noth U, Tuan RS. Concise review: the clinical application of mesenchymal stem cells for musculoskeletal regeneration: current status and perspectives. Stem Cells Transl Med. 2012 1(3):237-47.

35. Cui D, Li H, Xu X, Ye L, Zhou X, Zheng L, Zhou Y. Mesenchymal stem cells for cartilage regeneration of TMJ osteoarthritis. Stem Cells Int. 2017;2017: 5979741.

36. Pelletier JP, Martel-Pelletier J, Abramson SB. Osteoarthritis, an inflammatory disease: potential implication for the selection of new therapeutic targets. Arthritis Rheum. 2001:44(6):1237-47.

37. Guo JY, Li F, Wen YB, Cui HX, Guo ML, Zhang L, Zhang YF, Guo YJ, Guo YX. Melatonin inhibits Sirt1-dependent NAMPT and NFAT5 signaling in chondrocytes to attenuate osteoarthritis. Oncotarget. 2017:8(34):55967-83.

38. Hosseinzadeh A, Kamrava SK, Joghataei MT, Darabi R, Shakeri-Zadeh A, Shahriari M, Reiter RJ, Ghaznavi H, Mehrzadi S. Apoptosis signaling pathways in osteoarthritis and possible protective role of melatonin. J Pineal Res. 2016:61(4):411-25

39. Yang $X$, Shang H, Katz A, Li X. A modified aggregate culture for chondrogenesis of human adipose-derived stem cells genetically modified with growth and differentiation factor 5. BioResearch open access. 2013;2(4):258-65.
Ready to submit your research? Choose BMC and benefit from:

- fast, convenient online submission

- thorough peer review by experienced researchers in your field

- rapid publication on acceptance

- support for research data, including large and complex data types

- gold Open Access which fosters wider collaboration and increased citations

- maximum visibility for your research: over $100 \mathrm{M}$ website views per year

At BMC, research is always in progress.

Learn more biomedcentral.com/submissions 